\title{
Genetic Risk Factors for Major Bleeding in Patients Treated With Warfarin in a Community Setting
}

\author{
JA Roth ${ }^{1,2}$, D Boudreau ${ }^{1,2}$, MM Fujii $^{2}$, FM Farin ${ }^{3}$, AE Rettie $^{4}$, KE Thummel $^{5,6}$, and DL \\ Veenstra ${ }^{1,5}$ \\ ${ }^{1}$ Pharmaceutical Outcomes Research and Policy Program, Department of Pharmacy, University \\ of Washington, Seattle, Washington, USA \\ ${ }^{2}$ Group Health Research Institute, Group Health, Seattle, Washington, USA \\ ${ }^{3}$ Center for Ecogenetics and Environmental Health, University of Washington, Seattle, \\ Washington, USA \\ ${ }^{4}$ Department of Medicinal Chemistry, University of Washington, Seattle, Washington, USA \\ ${ }^{5}$ Institute for Public Health Genetics, University of Washington, Seattle, Washington, USA \\ ${ }^{6}$ Department of Pharmaceutics, University of Washington, Seattle, Washington, USA
}

\section{Abstract}

The influence of warfarin pharmacogenomics on major bleeding risk has been little studied in long-term users and non-specialist care settings. We conducted a case-control study to evaluate associations between $C Y P 2 C 9 * 2 / * 3, \operatorname{VKORC1}(1173)$, and $C Y P 4 F 2 * 3$ variants and major bleeding among patients treated with warfarin in a community setting. We calculated major bleeding odds ratios, adjusting for race, duration of warfarin use, age, gender, and body mass index. In 265 cases and 305 controls with 3.4 and 3.7 mean years of warfarin use, respectively, $C Y P 4 F 2 * 3$ was associated with decreased major bleeding risk (odds ratio: 0.62 ; $95 \%$ confidence interval: $0.43-$ 0.91). CYP2C $9 * 2 / * 3$ and VKORC1(1173) had null associations overall, but there was a nonsignificant increase in major bleeding risk in patients with duration $<6$ months (odds ratio: 1.30; $95 \%$ confidence interval: $0.60-2.83$; odds ratio: 1.23 ; $95 \%$ confidence interval: $0.57-2.64$, respectively). In summary, in the largest study of warfarin pharmacogenomics and major bleeding to date, we found a $38 \%$ lower risk in patients with $C Y P 4 F 2 * 3$, potentially reflecting interaction with warfarin and dietary vitamin $\mathrm{K}$ intake and warranting additional evaluation.

(C) 2014 American Society for Clinical Pharmacology and Therapeutics

Correspondence: DL Veenstra (veenstra@uw.edu).

SUPPLEMENTARY MATERIAL

Supplementary material is linked to the online version of the paper at http://www.nature.com/cpt AUTHOR CONTRIBUTIONS

J.A.R., D.L.V., D.B., and K.E.T. designed the research; J.A.R., D.B., M.M.F., F.M.F., and D.L.V. performed the research; J.A.R., D.B., and D.L.V. analyzed the data; J.A.R., D.B., M.M.F., F.M.F., K.E.T., A.E.R., and D.L.V. wrote the manuscript.

The authors declared no conflict of interest. 
Warfarin (Coumadin) is an anticoagulant that is highly effective at reducing thromboembolic event rates in patients with atrial fibrillation, stroke, joint replacement, and other conditions that confer increased clotting risk. ${ }^{1-4}$ However, warfarin therapy is complicated by a 20 -fold interpatient variation in therapeutic dose requirement and a related high rate of serious adverse bleeding events. These factors create barriers to the use of an agent with favorable benefit-risk balance in most indicated patients. ${ }^{5-10}$

The genomic advances of the past decade resulted in the discovery of three genes that significantly influence warfarin therapeutic dose requirement and may influence major bleeding risk. ${ }^{11,12}$ The cytochrome $\mathrm{P} 450$ 2C9 (CYP2C9) gene encodes a hepatic drugmetabolizing enzyme that is primarily responsible for metabolism of $S$-warfarin, the more active enantiomer of the racemic drug. ${ }^{13-15}$ Patients with the most common variants, CYP2C9*2 (rs17998523) and CYP2C9*3 (rs1057910), require significantly lower therapeutic warfarin doses relative to wild-type patients, and they are at an increased risk of major bleeding during the first year of warfarin use. ${ }^{12,16-18}$ The vitamin K epoxide reductase complex subunit 1 (VKORC1) gene encodes the target enzyme of warfarin. ${ }^{15,19-21}$ Patients with a variant $V K O R C 1$ allele $(1173 \mathrm{G}>\mathrm{A}$, rs 9934438$)$ require significantly lower warfarin doses vs. wild-type patients, and they may be at increased risk of major bleeding. ${ }^{19,21,22} \mathrm{On}$ the basis of this evidence, the warfarin label was revised to include information about CYP2C9 and VKORC1 testing in 2007. ${ }^{23}$ More recently, warfarin dose requirement has also been demonstrated to be associated with a variant of the cytochrome P450 4F2 (CYP4F2) gene. ${ }^{15,21,24,25}$ In several independent cohorts, patients with the $C Y P 4 F 2 * 3$ variant (rs2108622) required higher warfarin doses to achieve therapeutic anticoagulation levels (vs. wild-type patients). ${ }^{21,24-26}$ However, no studies to date have evaluated the association between $C Y P 4 F 2 * 3$ and the important clinical outcome of major bleeding. ${ }^{26}$

Although there is a relatively robust literature evaluating the impact of CYP2C9, VKORC1 and $C Y P 4 F 2$ polymorphisms on warfarin dose and treatment outcomes, most studies evaluated patients initiating warfarin in anticoagulation clinics at academic medical centers. ${ }^{13,17,18,22,27}$ Consequently, there is an evidence gap for long-term users and nonspecialist care settings, both of which are common among the estimated 2 million annual warfarin users in the United States. ${ }^{23}$ The recent introduction of new-generation oral anticoagulants (e.g., dabigatran, rivaroxaban, and apixaban) will probably decrease this number in coming years, but longitudinal market share data suggest that the pace of change will be slow, and warfarin will continue to be among the most commonly prescribed medications in the United States for some time. ${ }^{28,29}$ Thus, it remains important to investigate warfarin pharmacogenomic associations and appropriately translate key findings to enhance the benefit-risk balance of this highly effective and relatively inexpensive agent. In this study, we contribute to this effort by evaluating the association between CYP2C $9 * 2 / * 3$, VKORC1(1173), and CYP4F $2 * 3$ variants and major bleeding risk in patients treated with warfarin receiving care in an integrated health-care system spanning Washington state between 2005 and 2011. 


\section{RESULTS}

Among cases and controls, 50.6 and $57.7 \%$, respectively, were male; average age at the index date was 71.1 and 69.5 years, respectively; average duration of warfarin use at the index date was 3.4 and 3.7 years, respectively; and average time from the index date to survey date was 3.7 and 3.9 years, respectively. There were significant differences between cases and controls in the duration of warfarin therapy, heart valve replacement, atrial fibrillation, self-reported regular use of aspirin and vitamin $\mathrm{E}$, and diagnosis of congestive heart failure and hypertension (Table 1). There were not significant differences in any measured clinical or demographic covariates by CYP2C9, VKORC1, or CYP4F2 status. We found good agreement between our self-report survey variables and automated record. All study patients reported warfarin use, $94 \%$ reported starting warfarin prior to the index date, and $98 \%$ reported that an atrial fibrillation indication had corroborating medical record evidence. In addition, patients reporting excellent/very good, good, fair, and poor health at index date had mean Charlson Comorbidity Index scores of 1.03, 1.30, 1.89, and 2.10, respectively. The allele distributions for $C Y P 2 C 9 * 2$ and $* 3, V K O R C 11173 G>A$, and $C Y P 4 F 2 * 3$ did not significantly deviate from Hardy-Weinberg equilibrium (Supplementary Appendix C online). ${ }^{30}$

Univariate and multivariate analyses demonstrated significantly lower major bleeding risk in CYP4F2*3 variants relative to wild-type patients (univariate odds ratio (OR): $0.71 ; 95 \%$ confidence interval (CI): 0.51-0.98; multivariate OR: 0.62; 95\% CI: 0.43-0.91; Table 2). The CYP2C $* 2 / * 3$ and VKORC1(1173) variants had null associations with major bleeding in univariate and multivariate models (Table 2). The variant associations were similar in a subgroup analysis adjusting for international normalized ratio (INR) among patients in whom testing was done within 7 days before the index date (CYP4F2 OR: 0.51; 95\% CI: 0.28-0.93; CYP2C9 OR: 1.68; 95\% CI: 0.88-3.20; VKORC1 OR: 0.97; 95\% CI: 0.53-1.79) and within 30 days before the index date (CYP4F2 OR: 0.61; 95\% CI: 0.40-0.94; CYP2C9 OR: 1.03; 95\% CI: 0.66-1.60; VKORCI OR: 1.14; 95\% CI: 0.74-1.75).

In post hoc analyses of the association between clinical and demographic factors and major bleeding, heart valve replacement had the strongest major bleeding association (OR: 2.18; 95\% CI: 1.36-3.50). We also found significantly increased risk of major bleeding in patients with duration of therapy less than 90 days, 6 months, and 1 year (dichotomous variables), index age $>80$, congestive heart failure and hypertension diagnoses, and regular use of aspirin and vitamin $\mathrm{E}$ in the year prior to the index date (Figure 1). Patients with body mass index (BMI) 230 , atrial fibrillation indication, and anticoagulation clinic care had significantly reduced risk of major bleeding relative to those with $\mathrm{BMI}<30$, no atrial fibrillation indication, and non-specialist care, respectively (Figure 1).

We did not observe any statistically significant effects in post hoc analyses of the association between variants and major bleeding stratified by warfarin treatment duration, and the association between leafy green vegetable intake level and variability was stratified by variant status. However, all variants had stronger major bleeding associations in the first 6 months of warfarin therapy relative to patients with $>6$ months of use (Figure 1). The association between major bleeding and leafy green vegetable intake level and variability 
was similar across most genetic strata (Table 3). However, in both CYP4F2 analyses, variants demonstrated a protective trend (vs. wild type) against major bleeding associated with higher intake, and VKORCl variants demonstrated a trend toward higher major bleeding (vs. wild type) associated with higher intake.

\section{DISCUSSION}

We conducted a case-control study to investigate the associations between $C Y P 2 C 9 * 2 / * 3$, VKORC1(1173), and CYP4F2*3 variants and major bleeding in a community setting. We identified, validated, and collected genetic data on 265 patients with a major bleeding event while receiving warfarin, which is the largest number of major bleeding cases in a warfarin pharmacogenomics study to date. ${ }^{13,17,18,22}$ We found that a common $C Y P 4 F 2 * 3$ variant is associated with $\sim 40 \%$ lower major bleeding risk. By contrast, $C Y P 2 C 9 * 2 / * 3$ and VKORC1(1173) variants had null major bleeding associations in the primary analysis but showed a nonsignificant trend toward increased major bleeding risk in post hoc analyses of patients in the initial 6 months of warfarin use.

The validity of our findings is supported by several factors. First, the $C Y P 2 C 9 * 2 / * 3$, VKORC1(1173), and CYP4F $2 * 3$ allele frequency distributions were in Hardy-Weinberg equilibrium for both cases and controls, suggesting that there were not genotype ascertainment errors (Supplementary Appendix C online) ${ }^{30}$ Second, analyses restricted to patients with $<6$ months of warfarin use demonstrate $C Y P 2 C 9$ and VKORC1 major bleeding associations that are generally consistent with those of earlier studies evaluating similar time horizons. ${ }^{17,22}$ However, in this study, the CIs around the 6-month genetic associations are wide because the vast majority of the sample (78\%) used warfarin for more than 6 months (Table 1). Finally, we reproduced many clinical and demographic major bleeding associations that have been previously reported (Figure 1). ${ }^{17,31,32}$

\section{Implications}

Our findings are important because this is the first study to observe an association between the common $C Y P 4 F 2 * 3$ variant and clinically significant decreased risk of major bleeding. This protective effect is particularly interesting, given that patients with the variant require increased warfarin dose to achieve therapeutic INR levels. ${ }^{24,25}$ At the molecular level, this could be a consequence of diminished $C Y P 4 F 2 * 3$ function, resulting in lower capacity of the enzyme to metabolize vitamin $\mathrm{K}$, as well as better maintenance of the hepatic vitamin $\mathrm{K}$ cofactor needed to drive formation of clotting factors. Our exploratory analysis of the association between leafy green vegetable intake and major bleeding risk in $C Y P 4 F 2 * 3$ lends support to this hypothesis. In addition, we found that the protective effect of $C Y P 4 F 2 * 3$ is maintained well after the warfarin initiation phase, suggesting that it may offer some buffer against environmental changes that can trigger a major bleeding event. Again, at a mechanistic level, this could be the result of greater hepatic vitamin $\mathrm{K}$ reserve counteracting potential changes in dietary vitamin $\mathrm{K}$ intake, CYP2C9 or VKORCl function, or other factors that can increase major bleeding risk.

From a clinical perspective, our findings suggest that a "standard" warfarin dose may be less likely to over-anticoagulate patients with $C Y P 4 F 2 * 3$, thereby leading to reduced major 
bleeding risk. A similar argument has been previously advanced to account for findings that CYP2C9 variants were at increased risk of bleeding during warfarin initiation. In clinical practice, this information has the potential to benefit both $C Y P 4 F 2 * 3$ and wild-type patients. Testing for $C Y P 4 F 2 * 3$ status could potentially modify the benefit-risk trade-off of warfarin relative to new-generation anticoagulants (e.g., dabigatran, rivaroxaban, and apixaban), thus informing treatment selection. In addition, testing could identify wild-type patients at higher risk of major bleeding, helping clinicians to personalize monitoring intensity. Our findings should be validated in independent patient cohorts before $C Y P 4 F 2 * 3$ testing is used in order to inform treatment selection, dosing, or monitoring in routine clinical practice.

Our findings for $C Y P 2 C 9 * 2 / * 3$ and $V K O R C 1(1173)$ variants also have clinical relevance because they demonstrate that knowing CYP2C9 and VKORC1 genetic status is not likely to affect major bleeding risk after warfarin initiation. This finding is important, given that the warfarin package insert discusses the use of genetic testing to determine initial and maintenance dose, but it does not explicitly discuss test timing. ${ }^{23}$ In this context, our findings may be particularly useful to clinician, clinical guideline, and insurance formulary groups as they consider if, and when, warfarin genetic testing should be used in clinical practice.

Our results also suggest a variety of research questions to be addressed in future CYP4F2 studies. For example, we believe that additional studies should evaluate the association between variants and thromboembolic events, time to therapeutic INR, and time within therapeutic INR range. These studies should ideally leverage the strengths of prospective and retrospective designs to provide a comprehensive view of key cross-sectional and longitudinal health outcomes. This type of supplemental evidence could facilitate more robust assessment of the benefit-risk trade-offs of testing and could inform decisions about clinical use and reimbursement.

\section{Limitations}

This study has several important limitations. First, it should be noted that study patients were enrolled from a single integrated health-care system and may not be representative of other care settings or patient populations. This study is also limited by its retrospective study design because it prohibited inclusion of patients who were not alive to provide a survey and buccal swab. Accordingly, we do not consider major bleeding events that resulted in death, and therefore we may miss a small but clinically important subgroup. The survey results are limited because many patients were asked to recall information from several years in the past. This may have led to misclassification in some self-report items (e.g., intake of greens), and it should be considered when interpreting our exploratory analyses. However, we did find strong agreement between self-report and automated records for several variables. Another limitation is that pharmacy data idiosyncrasies limited our ability to precisely ascertain the duration of "current" episode of warfarin use. We evaluated alternative definitions based on 30-, 60-, and 120-day coverage gaps, but these approaches had little effect on the major bleeding odds ratios. Likewise, laboratory data idiosyncrasies and variable follow-up practices prohibited precise ascertainment of INR values at index dates. Instead, we used INR values within 7 and 30 days as proxies for values at the index date. 
Finally, we did not prespecify a Bonferroni correction to adjust the logistic regression a level for the three associations tested in the primary analysis. However, the $C Y P 4 F 2 * 3$ association remains significant even with this correction $(\alpha=0.017 ; P=0.014)$.

\section{Conclusions}

We identified a novel association between $C Y P 4 F 2 * 3$ and decreased risk of major bleeding in patients treated with warfarin and monitored in a community setting. We also found that CYP2C $9 * 2 / * 3$ and VKORC1(1173) variants did not confer increased major bleeding risk in the overall sample of predominately long-term users of warfarin and that both may have weaker major bleeding associations during initiation than those previously reported. Collectively, our findings expand the understanding of genetic risk factors for major bleeding in warfarin therapy and can potentially inform treatment selection, dosing, and monitoring practices pending validation in other community settings.

\section{METHODS}

\section{Study design}

We conducted a case-control study to investigate the association between $C Y P 2 C 9 * 2 / * 3$, VKORC1(1173), and CYP4F2*3 variants and major bleeding in patients receiving warfarin therapy in a community setting. The study was powered to evaluate the association between CYP2C9 variants and major bleeding, but we recruited all potential cases from the study period to enhance the statistical power to evaluate the association between VKORCl and CYP4F2 variants and major bleeding and to support stratified exploratory analyses. Target recruitment was 300 case patients and 300 control patients, which we estimated would provide $95 \%$ statistical power to evaluate the CYP2C9 association, assuming 30\% prevalence and a 2.0 major bleeding odds ratio for variants. ${ }^{17}$

All included patients were recruited in 2011 and were enrolled in the Group Health Cooperative $(\mathrm{GH})$, a nonprofit integrated health-care system that insures and provides comprehensive medical care for $\sim 650,000$ patients across Washington state. ${ }^{33} \mathrm{GH}$ maintains automated records of patient demographics, enrollment, inpatient and outpatient encounters, laboratory results, and pharmacy utilization, and a unique enrollee number links all records. Clinics owned by GH have had a fully integrated electronic medical record system that documents all patient-care contacts since 2005 . This study was approved by the GH and the University of Washington institutional review boards.

\section{Cases}

We identified GH patients with evidence of a major bleeding event between 1 January 2005 and 1 April 2011. We required major bleeding events to occur within warfarin supply (determined by pharmacy dispensing data) and included patients who met the following criteria at the index (bleeding) date: $\geq 18$ years of age, continuously enrolled in GH for $2+$ years prior to the index date, and no record of major bleeding in the year prior to the index date (Figure 2). We identified potential major bleeding events using a validated International Classification of Diseases, Ninth Revision algorithm with a positive predictive value of $87 \%$ (Supplementary Appendix A online). ${ }^{34}$ Trained abstractors validated potential 
bleeding events through electronic medical record review, and events were classified as "major bleeding" if (i) they were clinically overt and resulted in hospitalization, (ii) hemoglobin dropped $>2 \mathrm{mg} / \mathrm{dl}$, and/or (iii) $>2$ units of packed red blood cells were transfused. ${ }^{34,35}$ We also required bleeding events to be "definite," defined as documented visualization of blood by a physician, imaging demonstrating bleeding, and/or imaging of a bleeding source with physician-documented evidence of bleeding. ${ }^{34,36} \mathrm{We}$ excluded deceased and cognitively impaired cases that were identified during the medical record review. Our procedure identified 702 cases, and all were recruited for participation (Figure 2).

\section{Control patients}

We randomly selected one control for each case from the 27,928 patients with 1+ dispensing of warfarin during the study period (Figure 2). To do so, we used a risk set sampling approach in which we randomly assigned control patients an index date meeting the aforementioned eligibility criteria (except for a bleeding event at index), and then we selected controls from patients who were using warfarin and who were at risk for major bleeding at the time of case major bleeding events. ${ }^{37}$

Warfarin exposure ascertainment-We determined warfarin use from GH's automated pharmacy records. The evaluation of warfarin use included drug name, dispensing date, days of supply, and strength. An earlier study demonstrated excellent agreement with respect to warfarin use between GH's automated pharmacy data and medical charts. ${ }^{38}$ We calculated treatment duration based on the current episode of warfarin use, defined as continuous supply of warfarin with no more than 90 gap days. To calculate gap days, we used the days supply field from the dispensing data and multiplied it by 1.25 to account for variable adherence. ${ }^{39}$

\section{Genetic exposure ascertainment}

We collected a buccal swab from all study patients (catch-all mailer, Epicentre, Madison, WI) for analysis of $C Y P 2 C 9 * 2$ and $* 3$, VKORC1 $1173 G>A$, and $C Y P 4 F 2 * 3$ polymorphisms. The swab was mailed to the patients who consented to participation, along with the study survey described below. The polymorphism detection assays were designed and performed in the Functional Genomics Laboratory, Center for Ecogenetics and Environmental Health at the University of Washington, Seattle, WA. We extracted DNA from the buccal cell collection brushes using Qiagen kits and standard methods (Qiagen, Valencia, CA). We genotyped DNA samples using predesigned 5'-nuclease single-nucleotide polymorphism genotyping assays (Applied Biosystems, Foster City, CA), which employ specific fluorogenic probes. All probes were $3^{\prime}$-labeled with the TAMRA quencher dye. In addition, the specific wild-type and variant probes were $5^{\prime}$-labeled with the 6-FAM reporter dye and the VIC reporter dye, respectively.

The fluorescent 5'-nuclease assays were performed and analyzed on an ABI 7900HT Fast Real-Time PCR System (Applied Biosystems). The specific PCR reaction conditions were based on the general guidelines provided by the manufacturer and incorporated 10-25 ng of 
genomic DNA template. Thermocycling parameters consisted of an initial incubation at 95

${ }^{\circ} \mathrm{C}$ for $10 \mathrm{~min}$, followed by 50 cycles of $92{ }^{\circ} \mathrm{C}$ for $15 \mathrm{~s}$ and $60{ }^{\circ} \mathrm{C}$ for $90 \mathrm{~s}$.

We tested for deviation of genotype frequencies from Hardy-Weinberg equilibrium by applying the Hardy-Weinberg model to our data. ${ }^{30,40}$ We used the $\chi^{2}$ goodness-of-fit test to compare expected vs. actual prevalence of $C Y P 2 C 9, V K O R C 1$, and $C Y P 4 F 2$ alleles and defined significant deviation as a test resulting in $P<0.05{ }^{30}$

\section{Clinical, demographic, and behavioral covariates}

We obtained the clinical and demographic data elements shown in Table 1 from the GH automated databases. All study patients completed a 44-item self-report survey that provided supplemental information about clinical, demographic, and behavioral data elements that were not available in the GH automated databases. Table 1 shows the survey items utilized in this study, and the full survey is included in Supplementary Appendix B online. Among the 702 cases and 702 controls recruited for participation in the survey, 265 (38.0\%) and 305 (43.9\%) enrolled, and 437 (62.0\%) and 397 (56.1\%) refused participation or never responded, respectively. Participants and nonparticipants did not differ significantly by age ( $P=0.98)$, gender $(P=0.82)$, duration of GH enrollment $(P=0.93)$, or Charlson Comorbidity Index $(P=0.88)$.

\section{Statistical analysis}

We compared the characteristics of cases and controls using $\chi^{2}$ tests, Fisher's exact test for categorical variables (where appropriate), and the Student's $t$-test for continuous variables. We used logistic regression to estimate the risk of major bleeding associated with genetic variant status, expressed as univariate and multivariate ORs with respective CIs. In accordance with earlier studies, our analyses group heterozygous and homozygous variant patients in a single "variant" category due to the small number of homozygous variants. ${ }^{12,16,17,22,26,27}$

Although INR is probably part of the causal pathway between genetic status and risk of major bleeding, some earlier studies have adjusted for INR in statistical analyses. ${ }^{22,41} \mathrm{We}$ conducted subgroup analyses adjusting for INR level among patients with INR values within 7 days $(n=211)$ and 30 days $(n=393)$ of the index date to allow for better comparison with these studies. Many patients did not have INR documented precisely at the index date, so we used values from within 7 and 30 days as proxies for these values.

We also conducted post hoc validation and exploratory analyses to assess the associations between clinical and demographic covariates and major bleeding; genetic variant associations stratified by warfarin treatment duration; and self-reported leafy green vegetable intake level and variability associations stratified by genetic status. ${ }^{31,32,42-45} \mathrm{We}$ report all post hoc analyses as univariate associations and intend them for validation and exploratory purposes only. 


\section{Potential confounders}

Few of the covariates are associated with both the genetic variants and major bleeding risk.

For this reason, the only prespecified confounders were race/ethnicity and duration of warfarin therapy. We also evaluated warfarin indication, diagnosis of hypertension, diagnosis of congestive heart failure, and regular use of aspirin and vitamin $\mathrm{E}$ as potential confounders because they were significantly associated with case status, and they could have chance association with genetic status. We did not retain any of the post hoc potential confounders in the model because their inclusion resulted in $<5 \%$ change in all genetic variant coefficients.

We assessed all Table 1 covariates with biologically plausible association with major bleeding as potential precision variables. During this process, we used the Akaike Information Criterion to assess fit and select covariates to be retained in the model. ${ }^{46,47}$ The final multivariate logistic regression model included the following covariates: race (white/ other), duration of warfarin therapy (continuous), index age (continuous), gender, and BMI (continuous). All these covariates have been discussed as risk factors for major bleeding in earlier studies. ${ }^{31,32,48}$ We performed all analyses in STATA version 12.0 (STATA, Austin, TX).

\section{Supplementary Material}

Refer to Web version on PubMed Central for supplementary material.

\section{Acknowledgments}

This study was supported by grants from the National Institute of General Medical Sciences (1UO1 GM092676-01; K.E.T. and W. Burke, coprimary investigators), National Institute of Environmental Health Sciences (P30ES007033; D. Eaton, primary investigator), National Institute on Aging (T32 AG027677, A. LaCroix, primary investigator), and the PhRMA Foundation predoctoral fellowship in health outcomes (to J.A.R.). The funding sources had no role in the design or conduct of the study; collection, analysis, and interpretation of the data; preparation or review of the manuscript; or the decision to submit the manuscript for publication. Many thanks to the study participants; Kristin Delaney, Pat Janssen, Darren Malais, Jackie St. John, and Ellen Schartz for assistance with data collection, and Ann Wittkowsky for survey design input.

\section{References}

1. Go AS, et al. Anticoagulation therapy for stroke prevention in atrial fibrillation: how well do randomized trials translate into clinical practice? JAMA. 2003; 290:2685-2692. [PubMed: 14645310]

2. Agarwal S, Hachamovitch R, Menon V. Current trial-associated outcomes with warfarin in prevention of stroke in patients with nonvalvular atrial fibrillation: a meta-analysis. Arch Intern Med. 2012; 172:623-31. discussion 631. [PubMed: 22450212]

3. Salazar CA, Malaga G, Malasquez G. Direct thrombin inhibitors versus vitamin K antagonists or low molecular weight heparins for prevention of venous thromboembolism following total hip or knee replacement. Coch Database Syst Rev. 2010; 2010(4) CD005981.

4. Haq SA, Heitner JF, Sacchi TJ, Brener SJ. Long-term effect of chronic oral anticoagulation with warfarin after acute myocardial infarction. Am J Med. 2010; 123:250-258. [PubMed: 20193834]

5. Gurwitz JH, et al. Incidence and preventability of adverse drug events among older persons in the ambulatory setting. JAMA. 2003; 289:1107-1116. [PubMed: 12622580]

6. Hylek EM, Singer DE. Risk factors for intracranial hemorrhage in outpatients taking warfarin. Ann Intern Med. 1994; 120:897-902. [PubMed: 8172435] 
7. Fihn SD, Callahan CM, Martin DC, McDonell MB, Heniko JG, White RH. The risk for and severity of bleeding complications in elderly patients treated with warfarin. The National Consortium of Anticoagulation Clinics. Ann Intern Med. 1996; 124:970-979. [PubMed: 8624064]

8. Torn M, Cannegieter SC, Bollen WL, van der Meer FJ, van der Wall EE, Rosendaal FR. Optimal level of oral anticoagulant therapy for the prevention of arterial thrombosis in patients with mechanical heart valve prostheses, atrial fibrillation, or myocardial infarction: a prospective study of 4202 patients. Arch Intern Med. 2009; 169:1203-1209. [PubMed: 19597069]

9. Wysowski DK, Nourjah P, Swartz L. Bleeding complications with warfarin use: a prevalent adverse effect resulting in regulatory action. Arch Intern Med. 2007; 167:1414-1419. [PubMed: 17620536]

10. Baczek VL, Chen WT, Kluger J, Coleman CI. Predictors of warfarin use in atrial fibrillation in the United States: a systematic review and meta-analysis. BMC Fam Pract. 2012; 13:5. [PubMed: 22304704]

11. Gage BF, et al. Use of pharmacogenetic and clinical factors to predict the therapeutic dose of warfarin. Clin Pharmacol Ther. 2008; 84:326-331. [PubMed: 18305455]

12. Limdi NA, Veenstra DL. Expectations, validity, and reality in pharmacogenetics. J Clin Epidemiol. 2010; 63:960-969. [PubMed: 19995676]

13. Aithal GP, Day CP, Kesteven PJ, Daly AK. Association of polymorphisms in the cytochrome P450 CYP2C9 with warfarin dose requirement and risk of bleeding complications. Lancet. 1999; 353:717-719. [PubMed: 10073515]

14. Wadelius M, et al. Warfarin sensitivity related to CYP2C9, CYP3A5, ABCB1 (MDR1) and other factors. Pharmacogenomics J. 2004; 4:40-48. [PubMed: 14676821]

15. Takeuchi F, et al. A genome-wide association study confirms VKORC1, CYP2C9, and CYP4F2 as principal genetic determinants of warfarin dose. PLoS Genet. 2009; 5:e1000433. [PubMed: 19300499]

16. Limdi NA, et al. VKORC1 polymorphisms, haplotypes and haplotype groups on warfarin dose among African-Americans and European-Americans. Pharmacogenomics. 2008; 9:1445-1458. [PubMed: 18855533]

17. Higashi MK, et al. Association between CYP2C9 genetic variants and anticoagulation-related outcomes during warfarin therapy. JAMA. 2002; 287:1690-1698. [PubMed: 11926893]

18. Margaglione M, et al. Genetic modulation of oral anticoagulation with warfarin. Thromb Haemost. 2000; 84:775-778. [PubMed: 11127854]

19. Rieder MJ, et al. Effect of VKORC1 haplotypes on transcriptional regulation and warfarin dose. $\mathrm{N}$ Engl J Med. 2005; 352:2285-2293. [PubMed: 15930419]

20. Wadelius M, et al. Association of warfarin dose with genes involved in its action and metabolism. Hum Genet. 2007; 121:23-34. [PubMed: 17048007]

21. Wadelius M, et al. The largest prospective warfarin-treated cohort supports genetic forecasting. Blood. 2009; 113:784-792. [PubMed: 18574025]

22. Limdi NA, Wiener H, Goldstein JA, Acton RT, Beasley TM. Influence of CYP2C9 and VKORC1 on warfarin response during initiation of therapy. Blood Cells Mol Dis. 2009; 43:119-128. [PubMed: 19297219]

23. US Food and Drug Administration. FDA approves updated warfarin (coumadin) prescribing information. Aug 16.2007 Press release.

24. McDonald MG, Rieder MJ, Nakano M, Hsia CK, Rettie AE. CYP4F2 is a vitamin K1 oxidase: An explanation for altered warfarin dose in carriers of the V433M variant. Mol Pharmacol. 2009; 75:1337-1346. [PubMed: 19297519]

25. Caldwell MD, et al. CYP4F2 genetic variant alters required warfarin dose. Blood. 2008; 111:41064112. [PubMed: 18250228]

26. Bejarano-Achache I, Levy L, Mlynarsky L, Bialer M, Muszkat M, Caraco Y. Effects of CYP4F2 polymorphism on response to warfarin during induction phase: a prospective, open-label, observational cohort study. Clin Ther. 2012; 34:811-823. [PubMed: 22417713]

27. Lund K, Gaffney D, Spooner R, Etherington AM, Tansey P, Tait RC. Polymorphisms in VKORC1 have more impact than CYP2C9 polymorphisms on early warfarin International Normalized Ratio control and bleeding rates. Br J Haematol. 2012; 158:256-261. [PubMed: 22571356] 
28. Kirley K, Qato DM, Kornfield R, Stafford RS, Alexander GC. National trends in oral anticoagulant use in the United States, 2007 to 2011. Circ Cardiovasc Qual Outcomes. 2012; 5:615-621. [PubMed: 22949490]

29. IMS Institute for Healthcare Informatics. The use of medicines in the United States: review of 2011. 2012. <http://www.imshealth.com/ims/Global/Content/Insights/IMS\%20Institute\%20for \%20Healthcare\%20Informatics/IHII_Medicines_in_U.S_Report_2011.pdf>

30. Rodriguez S, Gaunt TR, Day IN. Hardy-Weinberg equilibrium testing of biological ascertainment for Mendelian randomization studies. Am J Epidemiol. 2009; 169:505-514. [PubMed: 19126586]

31. Palareti G, Cosmi B. Bleeding with anticoagulation therapy - who is at risk, and how best to identify such patients. Thromb Haemost. 2009; 102:268-278. [PubMed: 19652877]

32. Fang MC, et al. A new risk scheme to predict warfarin-associated hemorrhage: The ATRIA (Anticoagulation and Risk Factors in Atrial Fibrillation) Study. J Am Coll Cardiol. 2011; 58:395401. [PubMed: 21757117]

33. Saunders, KW.; Davis, RL.; Stergachis, A. Pharmacoepidemiology. Wiley; West Sussex, UK: 2005.

34. Arnason T, Wells PS, van Walraven C, Forster AJ. Accuracy of coding for possible warfarin complications in hospital discharge abstracts. Thromb Res. 2006; 118:253-262. [PubMed: 16081144]

35. White RH, Beyth RJ, Zhou H, Romano PS. Major bleeding after hospitalization for deep-venous thrombosis. Am J Med. 1999; 107:414-424. [PubMed: 10569295]

36. Cunningham A, Stein CM, Chung CP, Daugherty JR, Smalley WE, Ray WA. An automated database case definition for serious bleeding related to oral anticoagulant use. Pharmacoepidemiol Drug Saf. 2011; 20:560-566. [PubMed: 21387461]

37. Langholz B, Goldstein L. Risk set sampling in epidemiologic cohort studies. Statist Sci. 1996; 11(1):35-53.

38. Garg RK, et al. Ascertainment of warfarin and aspirin use by medical record review compared with automated pharmacy data. Pharmacoepidemiol Drug Saf. 2011; 20:313-316. [PubMed: 21351314]

39. Psaty RK, et al. The risk of myocardial infarction associated with antihypertensive drug therapies. JAMA. 1995; 274:620-625. [PubMed: 7637142]

40. Trikalinos TA, Salanti G, Khoury MJ, Ioannidis JP. Impact of violations and deviations in HardyWeinberg equilibrium on postulated gene-disease associations. Am J Epidemiol. 2006; 163:300309. [PubMed: 16410351]

41. Limdi NA, et al. Influence of CYP2C9 and VKORC1 1173C/T genotype on the risk of hemorrhagic complications in African-American and European-American patients on warfarin. Clin Pharmacol Ther. 2008; 83:312-321. [PubMed: 17653141]

42. Mantha S, Pianka AM, Tsapatsaris N. Determinants of intracranial hemorrhage incidence in patients on oral anticoagulation followed at the Lahey clinic. J Thromb Thrombolysis. 2011; 32:334-342. [PubMed: 21674132]

43. Hylek EM, Evans-Molina C, Shea C, Henault LE, Regan S. Major hemorrhage and tolerability of warfarin in the first year of therapy among elderly patients with atrial fibrillation. Circulation. 2007; 115:2689-2696. [PubMed: 17515465]

44. Palareti G, et al. Bleeding complications of oral anticoagulant treatment: an inception-cohort, prospective collaborative study (ISCOAT). Italian Study on Complications of Oral Anticoagulant Therapy. Lancet. 1996; 348:423-428. [PubMed: 8709780]

45. Lane $\mathrm{S}$, et al. The population pharmacokinetics of R- and S-warfarin: effect of genetic and clinical factors. Br J Clin Pharmacol. 2012; 73:66-76. [PubMed: 21692828]

46. Lindsey JK, Jones B. Choosing among generalized linear models applied to medical data. Stat Med. 1998; 17:59-68. [PubMed: 9463849]

47. Wagenmakers EJ, Farrell S. AIC model selection using Akaike weights. Psychon Bull Rev. 2004; 11:192-196. [PubMed: 15117008]

48. Apostolakis S, Lane DA, Guo Y, Buller H, Lip GY. Performance of the HEMORR(2)HAGES, ATRIA, and HAS-BLED bleeding risk-prediction scores in patients with atrial fibrillation undergoing anticoagulation: the AMADEUS (evaluating the use of SR34006 compared to warfarin 
or acenocoumarol in patients with atrial fibrillation) study. J Am Coll Cardiol. 2012; 60:861-867. [PubMed: 22858389] 


\section{Study Highlights}

\section{WHAT IS THE CURRENT KNOWLEDGE ON THE TOPIC?}

$\checkmark$ Studies evaluating new users of warfarin in anticoagulation clinics at academic medical centers demonstrate that $C Y P 2 C 9, V K O R C 1$, and $C Y P 4 F 2$ variants influence warfarin therapeutic dose requirements, and CYP2C9 and VKORC1 variants increase the risk of major bleeding.

\section{WHAT QUESTION DID THIS STUDY ADDRESS?}

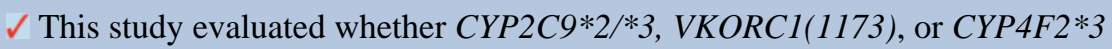
variants influence the risk of major bleeding in longer-term patients treated with warfarin cared for in a community setting.

\section{WHAT THIS STUDY ADDS TO OUR KNOWLEDGE}

$\checkmark$ We identified a novel association between $C Y P 4 F 2 * 3$ and decreased risk of major bleeding. We also found that $C Y P 2 C 9 * 2 / * 3$ and $V K O R C 1(1173)$ variants did not confer increased major bleeding risk in our predominately long-term users of warfarin, although variants in both genes may have a weaker association during initiation than those previously reported.

\section{HOW THIS MIGHT CHANGE CLINICAL PHARMACOLOGY AND THERAPEUTICS}

$\checkmark$ Our findings indicate a need to evaluate gene-drug-environment interactions in the study of warfarin and clinically relevant outcomes and can potentially inform treatment selection, dosing, and monitoring practices pending validation in other community settings. 


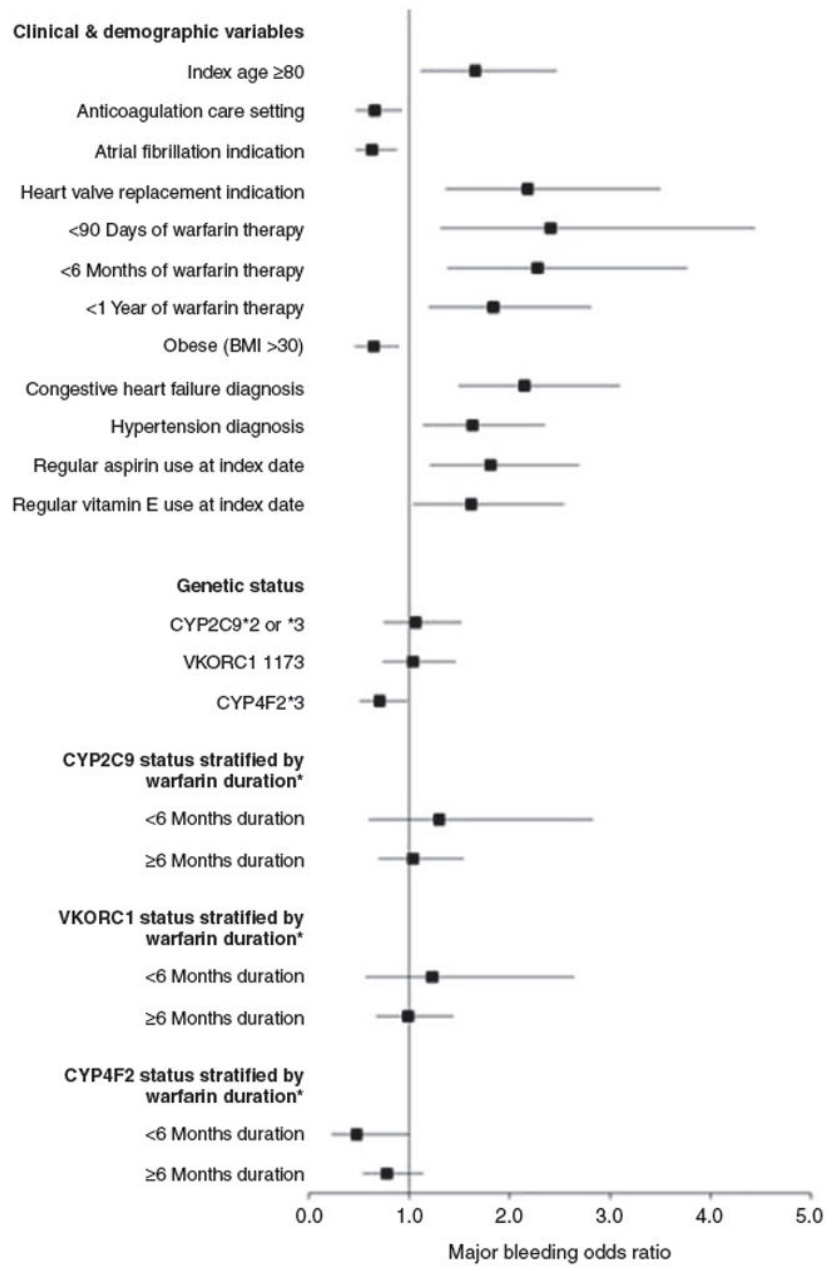

Figure 1.

Univariate major bleeding odds ratios by clinical, demographic, genetic, and warfarin duration-stratified subgroups. BMI, body mass index.

*Analysis compares odds of major bleeding in variants vs. wild-type patients within each strata of warfarin treatment duration. 


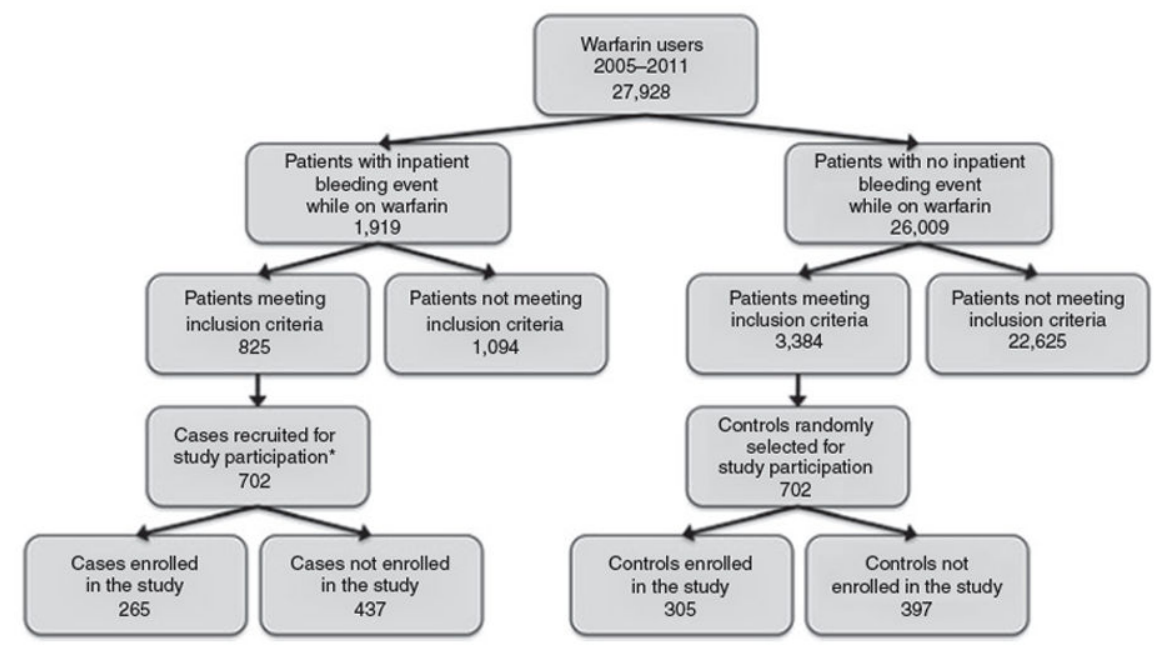

Figure 2.

Patient-selection flow diagram.

*Patients who were still alive and cognitively able to complete the survey at the time of study recruitment 
Table 1

Clinical and demographic covariates by case and control status

\begin{tabular}{|c|c|c|c|}
\hline Variable & Cases $(n=265)$ & Controls $(n=305)$ & $P$ value \\
\hline \multicolumn{4}{|l|}{ Automated data variables } \\
\hline Age in years at index date, mean $(\mathrm{SD})$ & $71.1(12.7)$ & $69.5(11.2)$ & 0.92 \\
\hline Duration of GH enrollment at index date (years), mean (SD) & $15.8(6.0)$ & $14.8(6.3)$ & 0.91 \\
\hline \multirow[t]{2}{*}{ International normalized ratio (INR) at index date, mean (SD) ${ }^{a}$} & $2.8(1.4)$ & $2.4(0.5)$ & 0.79 \\
\hline & \multicolumn{2}{|c|}{$n$ (column \%) } & \\
\hline Male & $134(50.6 \%)$ & $176(57.7 \%)$ & 0.09 \\
\hline BMI & & & 0.08 \\
\hline Underweight $(<18.5)$ & $1(0.3 \%)$ & $1(0.3 \%)$ & \\
\hline Normal weight (18.5-24.9) & $48(18.1 \%)$ & $42(13.8 \%)$ & \\
\hline Overweight (25.0-29.9) & $91(34.3 \%)$ & $85(27.9 \%)$ & \\
\hline Obese (>30.0) & $125(47.2 \%)$ & $177(58.0 \%)$ & \\
\hline Integrated group plan type & $214(80.8 \%)$ & $247(81.0 \%)$ & 0.95 \\
\hline Duration of warfarin therapy at index date & & & $<0.01$ \\
\hline$<6$ months & $69(26.0 \%)$ & $54(17.7 \%)$ & \\
\hline 6 months to 1 year & $26(9.8 \%)$ & $24(7.9 \%)$ & \\
\hline$>1$ year & $170(64.2 \%)$ & $227(74.4 \%)$ & \\
\hline Patient residence in metropolitan setting & $218(82.3 \%)$ & $246(80.7 \%)$ & 0.62 \\
\hline Reside $>10$ miles from warfarin-monitoring provider & $51(21.3 \%)$ & $59(20.2 \%)$ & 0.75 \\
\hline \multicolumn{4}{|l|}{ Comorbidities } \\
\hline Cancer & $8(3.0 \%)$ & $14(4.6 \%)$ & 0.32 \\
\hline Diabetes & $58(21.9 \%)$ & $81(26.6 \%)$ & 0.19 \\
\hline Hypertension & $197(74.3 \%)$ & $195(63.9 \%)$ & $<0.01$ \\
\hline Congestive heart failure & $101(38.1 \%)$ & $68(22.3 \%)$ & $<0.01$ \\
\hline Charlson Comorbidity Index, mean (SD) & $1.6(1.7)$ & $1.3(1.6)$ & 0.90 \\
\hline \multicolumn{4}{|l|}{ Self-reported survey variables } \\
\hline \multicolumn{4}{|c|}{$n$ (column \%) } \\
\hline \multicolumn{4}{|l|}{ Warfarin indication } \\
\hline Atrial fibrillation & $89(33.6 \%)$ & $136(44.6 \%)$ & $<0.01$ \\
\hline DVT & $31(11.7 \%)$ & $42(13.8 \%)$ & 0.45 \\
\hline $\mathrm{PE}$ & $27(10.2 \%)$ & $26(8.5 \%)$ & 0.49 \\
\hline Stroke & $27(10.2 \%)$ & $20(6.6 \%)$ & 0.12 \\
\hline Heart valve replacement & $54(20.4 \%)$ & $32(10.5 \%)$ & 0.01 \\
\hline Myocardial infarction & $13(4.9 \%)$ & $17(5.6 \%)$ & 0.71 \\
\hline Joint replacement & $12(4.5 \%)$ & $12(3.9 \%)$ & 0.72 \\
\hline CABG & $6(2.3 \%)$ & $11(3.6 \%)$ & 0.36 \\
\hline Other & $17(6.4 \%)$ & $23(7.5 \%)$ & 0.61 \\
\hline
\end{tabular}




\begin{tabular}{|c|c|c|c|}
\hline Variable & Cases $(n=265)$ & Controls $(n=305)$ & $P$ value \\
\hline Race & & & 0.45 \\
\hline American Indian or Alaska Native & $3(1.1 \%)$ & $1(0.3 \%)$ & \\
\hline Asian & $3(1.1 \%)$ & $6(2.0 \%)$ & \\
\hline Black/African American & $7(2.7 \%)$ & $5(1.7 \%)$ & \\
\hline White/Caucasian & $243(93.1 \%)$ & $287(95.0 \%)$ & \\
\hline Other & $4(1.5 \%)$ & $2(0.6 \%)$ & \\
\hline Care setting & & & 0.12 \\
\hline Anticoagulation clinic & $115(43.4 \%)$ & $166(54.4 \%)$ & \\
\hline Primary care & $86(32.5 \%)$ & $91(29.8 \%)$ & \\
\hline Cardiologist & $20(7.5 \%)$ & $24(7.9 \%)$ & \\
\hline Other & $27(10.2 \%)$ & $20(6.6 \%)$ & \\
\hline \multicolumn{4}{|l|}{ Concomitant OTC medications } \\
\hline NSAID & $22(10.2 \%)$ & $13(4.3 \%)$ & 0.07 \\
\hline Acetaminophen & $74(32.6 \%)$ & $85(28.1 \%)$ & 0.24 \\
\hline Aspirin & $70(32.6 \%)$ & $63(21.1 \%)$ & $<0.01$ \\
\hline Vitamin E & $49(23.4 \%)$ & $47(15.9 \%)$ & 0.02 \\
\hline Leafy green vegetable intake level & & & 0.78 \\
\hline$\unlhd$ Serving per week & $133(53.8 \%)$ & $167(55.3 \%)$ & \\
\hline 2-3 Servings per week & $72(29.1 \%)$ & $91(30.1 \%)$ & \\
\hline 4-6 Servings per week & $24(9.7 \%)$ & $30(9.9 \%)$ & \\
\hline 1 Serving per day & $17(6.9 \%)$ & $13(4.3 \%)$ & \\
\hline$>1$ Serving per day & $1(0.4 \%)$ & $1(0.3 \%)$ & \\
\hline Leafy green vegetable intake variability & & & 0.89 \\
\hline Variable leafy green vegetable intake & $44(17.7 \%)$ & $51(16.9 \%)$ & \\
\hline Constant leafy green vegetable intake & $204(82.3 \%)$ & $251(83.1 \%)$ & \\
\hline
\end{tabular}

Bold $P$ values indicate statistically significant differences between cases and controls at $\mathrm{a}=0.05$.

BMI, body mass index; CABG, coronary artery bypass graft; DVT, deep vein thrombosis; GH, Group Health; NSAID, nonsteroidal antiinflammatory drug; OTC, over-the-counter; PE, pulmonary embolism.

${ }^{a}$ Among patients with an INR value recorded within 30 days before the index date. 


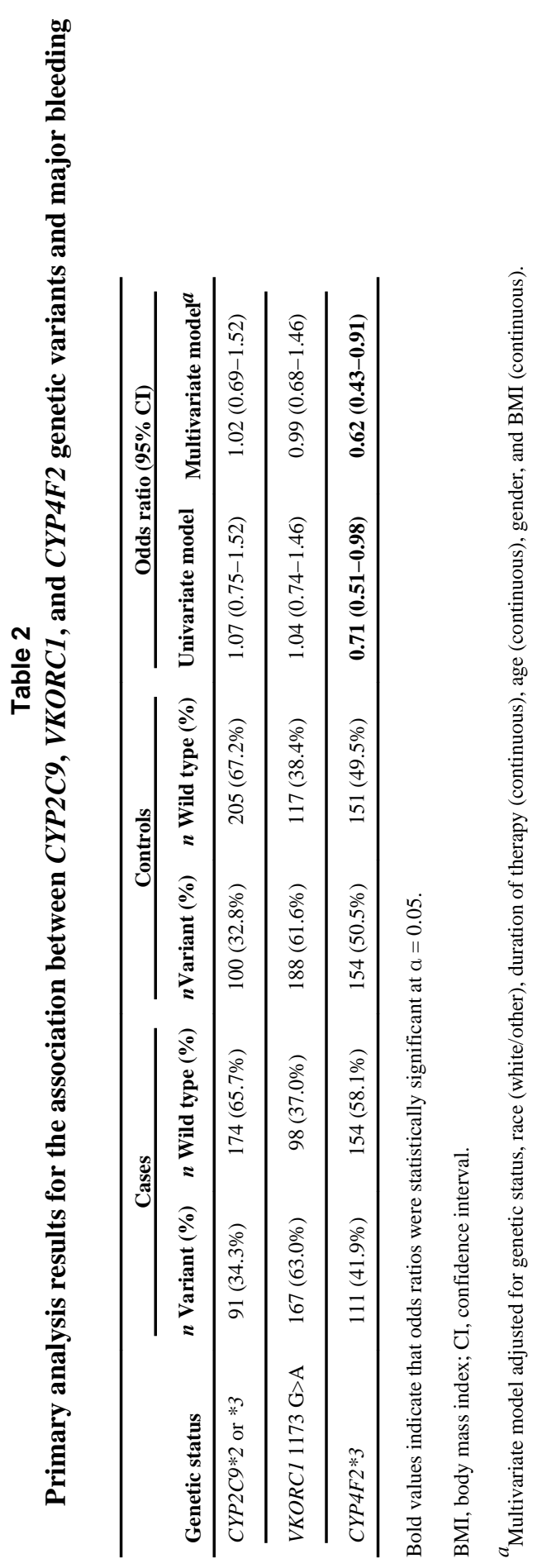


Table 3

Exploratory analysis of the effect of genetic status on dietary major bleeding risk

\begin{tabular}{lcc}
\hline Genetic status & 2+ vs. $\mathbf{4}$ Servings of leafy greens/week, or $\mathbf{9 5 \%} \mathbf{C I})$ & $\begin{array}{c}\text { Variable vs. constant leafy green vegetable intake by } \\
\text { week, or } \mathbf{9 5 \%} \text { CI) }\end{array}$ \\
\hline CYP2C9 wild type & $1.02(0.67-1.56)$ & $1.05(0.61-1.81)$ \\
\hline CYP2C $9 * 2$ or *3 & $1.11(0.62-1.96)$ & $1.08(0.50-2.34)$ \\
\hline VKORC1 wild type & $0.74(0.43-1.29)$ & $1.09(0.51-2.29)$ \\
\hline VKORC1 1173 G>A & $1.32(0.86-2.03)$ & $1.04(0.60-1.81)$ \\
\hline CYP4F2 wild type & $1.15(0.73-1.83)$ & $1.18(0.64-2.16)$ \\
\hline CYP4F $2 * 3$ & $0.96(0.58-1.58)$ & $0.95(0.49-1.82)$ \\
\hline
\end{tabular}

These analyses are grouped by genetic status (left column) and evaluate the major bleeding odds ratio for patients with high leafy green vegetable intake levels vs. those with low leafy green vegetable intake levels (center column) and patients with variable leafy green vegetable intake vs. those with constant leafy green vegetable intake (right column). None of the odds ratios above were statistically significant at $\mathrm{\alpha}=0.05$.

CI, confidence interval; OR, odds ratio. 